\title{
Clinical Outcomes of 67 Patients Treated with Chemoradiotherapy for Primary Thyroid Non-Hodgkin's Lymphoma in Osaka Medical College
}

\author{
Tsuyoshi Komori*\#, Isamu Narabayashi”, Yoshifumi Narumi, Taisuke Inomata \\ Department of Radiology, Osaka Medical College, Takatsuki, Japan \\ Email: "komoyan1@gmail.com
}

Received 28 February 2016; accepted 2 May 2016; published 5 May 2016

Copyright (C 2016 by authors and Scientific Research Publishing Inc.

This work is licensed under the Creative Commons Attribution International License (CC BY). http://creativecommons.org/licenses/by/4.0/

(c) (i) Open Access

\section{Abstract}

Background: The reports of clinical outcomes of patients treated with chemoradiotherapy for primary thyroid non-Hodgkin's lymphoma are rare. We report our results of chemoradiotherapy for primary thyroid non-Hodgkin's lymphoma. Materials and Methods: The subjects were 67 patients with thyroid non-Hodgkin's lymphoma among 269 patients with malignant lymphoma who received radiotherapy in our hospital during a period between May 1990 and June 2005. The patients included 16 men and 51 women, with a mean age of $66.2 \pm 10.7$ years (30 - 84 years). The disease stage was stage $I$ in 42 patients, stage II in 24, and unclear in 1 . The histologic type was B-cell lymphoma in 66 patients, MALT in 9, diffuse type in 52, follicular type in 5, and diffuse and follicular type in 1. CHOP chemotherapy regimen for malignant lymphoma patients was as follows. Intravenous drip infusion of cyclophosphamide $750 \mathrm{mg} / \mathrm{m}^{2}$, (drip) infusion of doxorubicin 50 $\mathrm{mg} / \mathrm{m}^{2}$, and intravenous injection of vincristine $1.4 \mathrm{mg} / \mathrm{m}^{2}$ were administered on day 1 , followed by 5 consecutive days of oral prednisolone $100 \mathrm{mg} / \mathrm{m}^{2}$. This regimen was repeated every 3 weeks (21 days) in 6 to 8 courses. Modified CHOP chemotherapy regimen was as follows. Intravenous drip infusion of cyclophosphamide $600 \mathrm{mg} / \mathrm{m}^{2}$, intravenous (drip) infusion of doxorubicin $40 \mathrm{mg} / \mathrm{m}^{2}$, intravenous infusion of vindesine $3 \mathrm{mg} / \mathrm{m}^{2}$, and intravenous drip infusion of prednisolone 60 $\mathrm{mg} /$ body were administered on day 1 , and intravenous prednisolone was changed to oral prednisolone with the dose tapered gradually. After completing one course of this regimen, two courses of radiotherapy (a total of $36 \mathrm{~Gy}$ ) were performed, followed by 6 courses of the chemotherapy regimen at lower doses ( $80 \%$ of the initial doses) repeated once a month. Results: Results of chemoradiotherapy in all patients were excellent. The 15 -year survival rate was over $80 \%$. Although there were no significant differences in the results of chemoradiotherapy among different histo-

\footnotetext{
"Corresponding author.

"Tsuyoshi Komori and Isamu Narabayashi were equally contributed in this study.
}

How to cite this paper: Komori, T., Narabayashi, I., Narumi, Y. and Inomata, T. (2016) Clinical Outcomes of 67 Patients Treated with Chemoradiotherapy for Primary Thyroid Non-Hodgkin's Lymphoma in Osaka Medical College. Journal of Cancer Therapy, 7, 329-334. http://dx.doi.org/10.4236/jct.2016.75035 
logic types of thyroid malignant lymphoma, the survival rate was $100 \%$ for MALT type, as compared with poor results for diffuse large type or diffuse mixed type. The analysis of the results of chemoradiotherapy according to the stage of malignant thyroid lymphomas revealed that therapeutic results were significantly better in stage I than in stage II. Conclusion: The CHOP chemoradiotherapy regimen and modified CHOP chemoradiotherapy regimen were excellent for primary thyroid non-Hodgkin's lymphoma.

\section{Keywords}

Thyroid Non-Hodgkin's Lymphoma, Chemoradiotherapy

\section{Introduction}

Malignant lymphoma is a collective term for malignant neoplasms of lymphoid tissue caused by proliferation of lymphocytes. The incidence of malignant lymphoma in Japan has been increasing with aging of the population. Three mechanisms underlying the development of this malignancy are considered as follows: gene abnormalities that cause an increase in cell proliferation, prolongation of cellular life span due to inhibition of apoptosis by overexpression of BCL-2 gene, and involvement of exogenous factors such as viruses and bacteria.

A diagnosis of malignant lymphoma basically depends on pathological evaluation of tissue biopsies, and the new WHO classification of malignant lymphoma has been used since 2008 (Table 1). This classification is based on the Revised European American Lymphoma (REAL) classification published in 1994. Modified classification of lymphomas is attributable to an increased knowledge of kinds of monoclonal antibodies identifying the immune surface phenotype of lymphocytes and molecular genetics of lymphomas. Malignant lymphomas are roughly classified into Hodgkin's lymphomas and non-Hodgkin's lymphomas, and the WHO classification includes more than 20 subtypes of non-Hodgkin's lymphomas. More specifically, non-Hodgkin's lymphomas are firstly classified as precursor cell-type, B cell-type, or T cell-type, and are further subclassified in terms of cell morphology and immune surface materials based on chromosomal analysis and molecular genetic analysis. Diffuse Large B-Cell Lymphoma (DLBCL) and follicular lymphomas account for $50 \%$ of all malignant lymphomas. DLBCL with expression of CD10 is known to have a poor prognosis.

\section{Etiology of Malignant Lymphomas}

Overexpression of c-myc oncogene on chromosome 8 and Cyclin D1 gene facilitates cell proliferation, and overexpression of Bcl-2 gene that causes Burkitt's lymphoma and mantle cell lymphoma leads to prolongation of cellular life span due to inhibition of apoptosis and causes follicular lymphoma.

Exogenous factors include viruses and bacteria. In Hodgkin's lymphoma, Epstein-Barr virus is found in RS cells, and this is reported to be the cause of the disease.

\section{Classification of Malignant Lymphomas}

Malignant lymphomas are divided into two groups: Hodgkin's lymphoma and non-Hodgkin's lymphoma. The incidence of Hodgkin's lymphomas in Japan is $4 \%-5 \%$, which is lower than the corresponding rates of $40 \%$ $50 \%$ in Europe and the United States. The incidence of non-Hodgkin's lymphomas is about $95 \%$, combining B-cell lymphomas and T-cell lymphomas. Hodgkin's lymphoma was first reported by Hodgkin in UK in 1832, and called Hodgkin's disease. Non-Hodgkin's disease was distinguished from Hodgkin's disease around 1900. Virchow used the term lymphosarcoma in 1863.

The incidence of Hodgkin's lymphoma is decreasing annually. This might be explained by a definitive diagnosis of non-Hodgkin's lymphomas due to advances in the techniques of pathological diagnosis, which had been misdiagnosed as Hodgkin's lymphoma.

Non-Hodgkin's lymphomas were classified by the National Cancer Institute Working Formulation (WF) classification in the US in the 1980s, and the REAL classification began to be used in 1994. Since 2008, the WHO classification (Table 1) has been used worldwide as the standard classification of lymphoid neoplasms [1] [2]. Modified classification of lymphomas from the WF classification and the REAL classification to the WHO 
Table 1. WHO 2008: the mature B-cell neoplasms.

Chronic lymphocytic leukemia/small lymphocytic lymphoma

B-cell prolymphocytic leukemia

Splenic marginal zone lymphoma

Hairy cell leukemia

Splenic lymphoma/leukemia, unclassifiable

Splenic diffuse red pulp small B-cell lymphoma*

Hairy cell leukemia-variant ${ }^{*}$

Lymphoplasmacytic lymphoma

Waldenström macroglobulinemia

Heavy chain diseases

Alpha heavy chain disease

Gamma heavy chain disease

Mu heavy chain disease

Plasma cell myeloma

Solitary plasmacytoma of bone

Extraosseous plasmacytoma

Extranodal marginal zone B-cell lymphoma of mucosa-associated lymphoid tissue (MALT lymphoma)

Nodal marginal zone B-cell lymphoma (MZL)

Pediatric type nodal MZL

Follicular lymphoma

Pediatric type follicular lymphoma

Primary cutaneous follicle center lymphoma

Mantle cell lymphoma

Diffuse large B-cell lymphoma (DLBCL), not otherwise specified

$\mathrm{T}$ cell/histiocyte rich large B-cell lymphoma

$D L B C L$ associated with chronic inflammation

Epstein-Barr virus $(E B V)+D L B C L$ of the elderly

Lymphomatoid granulomatosis

Primary mediastinal (thymic) large B-cell lymphoma

Intravascular large B-cell lymphoma

Primary cutaneous DLBCL, leg type

ALK + large B-cell lymphoma

Plasmablastic lymphoma

Primary effusion lymphoma

Large B-cell lymphoma arising in HHV8-associated multicentric

Castleman disease

Burkitt lymphoma

B-cell lymphoma, unclassifiable, with features intermediate

between diffuse large B-cell lymphoma and Burkitt lymphoma

B-cell lymphoma, unclassifiable, with features intermediate between

diffuse large B-cell lymphoma and classical Hodgkin lymphoma

Hodgkin Lymphoma

Nodular lymphocyte-predominant Hodgkin lymphoma

Classical Hodgkin lymphoma

Nodular sclerosis classical Hodgkin lymphoma

Lymphocyte-rich classical Hodgkin lymphoma

Mixed cellularity classical Hodgkin lymphoma

Lymphocyte-depleted classical Hodgkin lymphoma

${ }^{*}$ These represent provisional entities or provisional subtypes of other neoplasms. Diseases shown in italics are newly included in the 2008 WHO. 
classification was attributable to an increased knowledge of the types of monoclonal antibodies that identify the immune surface phenotype of lymphocytes and the progress in molecular genetics of lymphomas, but there always seems to be a difference in the viewpoint between pathologists who are in charge of classification and clinicians who places much value on prognostication.

The WHO classification is advantageous in that it reflects clinical characteristics of lymphomas and subclassifies morphology, antigenicity, and genetics in a consistent manner. It is speculated that DLBCL, the most frequent type of lymphoma, is comprised of multiple subtypes with different prognoses, and its subclassification is now under consideration. It has become apparent that CD10 expression in DLBCL is associated with a poor prognosis.

\section{Treatments of Malignant Lymphomas}

1) Radiotherapy (external irradiation: Mantle/inverted Y, etc.)

2) Chemotherapy (anticancer agents: CHOP, modified CHOP, fludarabine phosphate)

3) Biological products: anti-CD20 antibody (Rituxan)

4) Follow-up observation (watchful waiting, careful observation)

5) Hematopoietic stem cell transplantation: autologous transplantation, allogeneic transplantation

6) Radioimmunotherapy (Zevalin, Bxxar)

\section{Patients with Thyroid Non-Hodgkin's Lymphoma Treated in Osaka Medical College}

The subjects were 67 patients with thyroid non-Hodgkin’s lymphoma among 269 patients with malignant lymphoma who received radiotherapy in our hospital during a period between May 1990 and June 2005. The patients included 16 men and 51 women, with a mean age of $66.2 \pm 10.7$ years ( 30 - 84 years). The disease stage was stage I in 42 patients, stage II in 24, and unclear in 1. The histologic type was B-cell lymphoma in 66 patients, MALT in 9, diffuse type in 52, follicular type in 5, and diffuse and follicular type in 1.

CHOP chemotherapy regimen for malignant lymphoma patients was as follows. Intravenous drip infusion of cyclophosphamide $750 \mathrm{mg} / \mathrm{m}^{2}$, (drip) infusion of doxorubicin $50 \mathrm{mg} / \mathrm{m}^{2}$, and intravenous injection of vincristine $1.4 \mathrm{mg} / \mathrm{m}^{2}$ were administered on day 1 , followed by 5 consecutive days of oral prednisolone $100 \mathrm{mg} / \mathrm{m}^{2}$. This regimen was repeated every 3 weeks (21 days) in 6 to 8 courses.

Modified CHOP chemotherapy regimen was as follows. Intravenous drip infusion of cyclophosphamide 600 $\mathrm{mg} / \mathrm{m}^{2}$, intravenous (drip) infusion of doxorubicin $40 \mathrm{mg} / \mathrm{m}^{2}$, intravenous infusion of Vindesine $3 \mathrm{mg} / \mathrm{m}^{2}$, and intravenous drip infusion of prednisolone $60 \mathrm{mg} /$ body were administered on day 1 , and intravenous prednisolone was changed to oral prednisolone with the dose tapered gradually. After completing one course of this regimen, two courses of radiotherapy (a total of $36 \mathrm{~Gy}$ ) were performed, followed by 6 courses of the chemotherapy regimen at lower doses ( $80 \%$ of the initial doses) repeated once a month.

Figure 1 shows the results of chemoradiotherapy for cases of thyroid non-Hodgkin's lymphoma treated in Osaka Medical College. These results were excellent.

Although there were no significant differences in the results of chemoradiotherapy among different histologic types of thyroid malignant lymphoma, the survival rate was 100\% for MALT type, as compared with poor results for diffuse large type or diffuse mixed type (Figure 2).

As shown in Figure 3, the analysis of the results of chemoradiotherapy according to the stage of malignant thyroid lymphomas revealed that therapeutic results were significantly better in stage I than in stage II.

As for the results of radiography for malignant thyroid lymphomas treated in Osaka Medical College according to the pre-therapeutic IL-2R value, the survival rate was $100 \%$ when the IL-2R value was $530 \mathrm{U} / \mathrm{ml}$ or less, showing a tendency to better outcome with IL-2R values of $531 \mathrm{U} / \mathrm{ml}$ or more. As for the LDH value, the results of chemoradiotherapy were significantly better in those with LDH values of $2501 \mathrm{U} / \mathrm{L}$ or less than those with higher LDH values.

\section{Discussion and Conclusion}

In Osaka Medical College, ${ }^{67}$ Ga scintigraphy or FDG-PET has been performed to evaluate the staging, and modified CHOP chemotherapy has always been administered even when radiotherapy was requested. Treatment of malignant lymphomas performed in Osaka Medical College has also been reported frequently in the literature [3]-[5]. 


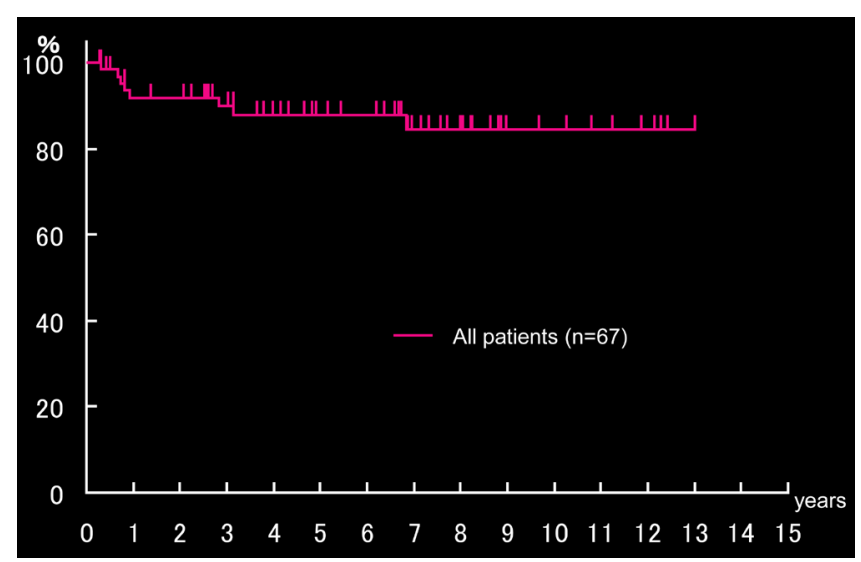

Figure 1. Patients with malignant thyroid lymphoma treated in Osaka Medical College: Results of chemoradiotherapy in all patients.

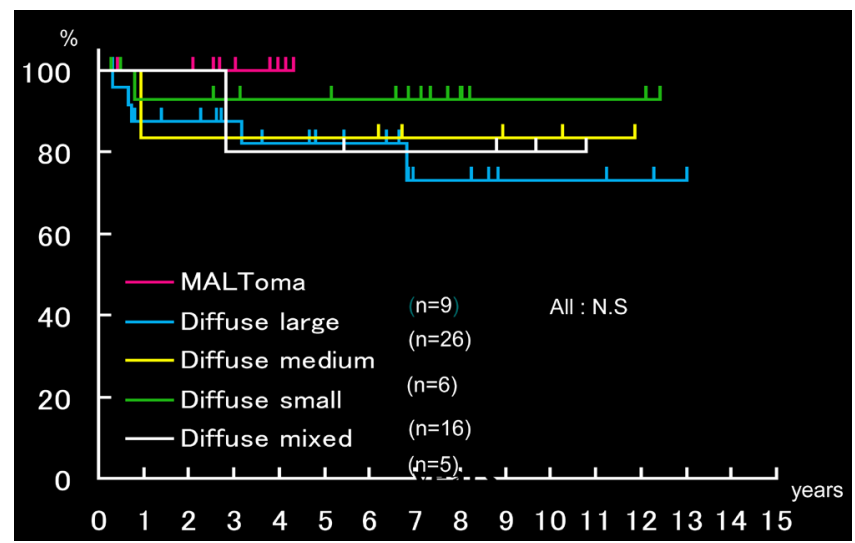

Figure 2. Patients with malignant thyroid lymphoma treated in Osaka Medical College: Results of chemoradiotherapy by disease stage.

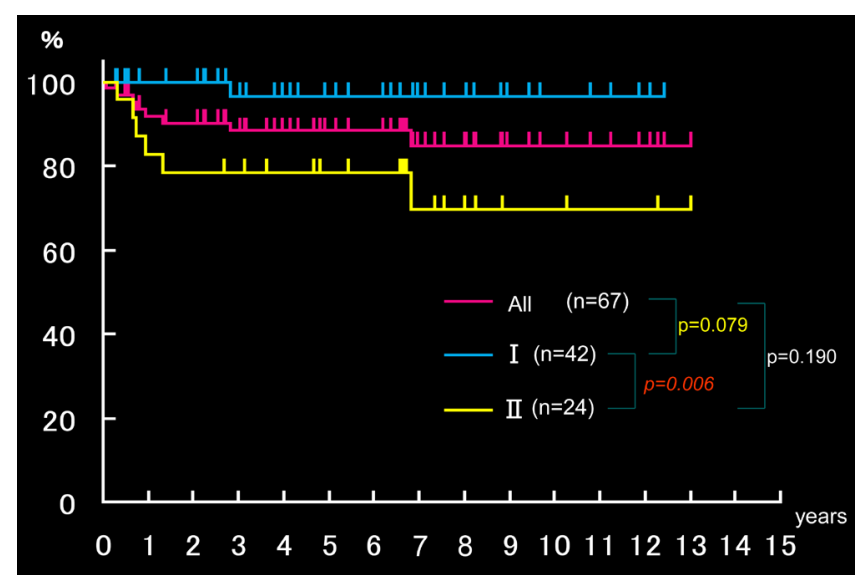

Figure 3. Patients with malignant thyroid lymphoma treated in Osaka Medical College: Results of chemoradiotherapy by histologic type.

Patients with malignant lymphoma have been increasing with aging of the society, and therefore, preparation of guidelines for staging by diagnostic imaging using PET with FDG, CT, or MRI, evaluation of therapeutic results, and diagnosis of recurrence is required. In the beginning, PET/CT apparatus, which is indispensable for disease staging, evaluation of therapeutic results, and diagnosis of recurrence, was not available at Osaka Medi- 
cal College, but it was introduced in 2014. If a schedule for combined use of R-CHOP and radiotherapy is available at the time when the diagnosis is established, it would improve therapeutic outcomes. It is essential to cooperate between radiologists (diagnostic radiologists, radiation oncologists) and medical oncologists.

The clinical utility of radioimmunotherapy, employing nuclear medicine technology, is encouraging. In particular, it can help to improve prognosis in patients with stage III or IV malignant lymphomas or with recurrent disease. The use of Zevalin is considered overseas as a first-line therapy or high-dose therapy. This drug appears to be promising for non-Hodgkin's lymphomas; in particular, dramatic improvement is awaited not only for progressive malignant lymphomas of moderate to high malignancy but also for indolent non-Hodgkin's lymphomas of low malignancy.

Dosimetry of ${ }^{90}$ Y-labeled anti-CD20 monoclonal antibody (Zevalin) in malignant lymphoma tissue is a future topic of discussion.

When external irradiation using appropriate irradiation field by scintigraphy (Ga-67 citrate and F-18 FDG) is used, a dose of 36 Gy should be delivered as a preferred dose to prevent the recurrence of malignant lymphoma. A dose of $30 \mathrm{~Gy}$ is sufficient for regional lymph nodes when there is no evidence of mass on imaging. For solid epithelial cancer, a dose of 70 - 80 Gy is necessary, which suggests that there are few clinical expectations of radioimmunotherapy for the treatment of solid cancer.

\section{Acknowledgements}

We gratefully acknowledge the secretary assistance of Ms. Naomi Komashita and Ms. Chieko Hashimoto.

\section{Conflict of Interest Statement}

None.

\section{References}

[1] (1982) National Cancer Institute Sponsored Study of Classifications of Non-Hodgkin's Iymphomas: Summary and Description of a Working Formulation for Clinical Usage. The Non-Hodgkin’s Lymphoma Pathologic Classification Project. Cancer, 49, 2112-2135.

http://dx.doi.org/10.1002/1097-0142(19820515)49:10<2112::AID-CNCR2820491024>3.0.CO;2-2

[2] (1993) The Intemational Non-Hodgkin's Iymphoma Prognostic Factors Project: A Predictive Model for Aggressive Non-Hodgkin's Iymphoma. New England Journal of Medicine, 329, 987-994. http://dx.doi.org/10.1056/NEJM199309303291402

[3] Matsuzuka, F., Miyauchi, A., Katayama, S., Narabayashi, I., Ikeda, H., Kuma, K. and Sugawara, M. (1993) Clinical Aspects of Primary Thyroid Lymphoma: Diagnosis and Treatment Based Our Experience of 119 Cases. Thyroid, 3, 93-99. http://dx.doi.org/10.1089/thy.1993.3.93

[4] Yuki, M., Narabayashi, I., Yamamoto, K., Shimizu, T., Tanaka, Y., Tatsumi, T. and Komori, T. (2000) Multifocal Primaly Lymphoma of Bone Scintigraphy and MR Findings before and after Treatment. Radiation Medicine, 18, 305310.

[5] Takakuwa, T., Hongyo, T., Syaifudin, M., Kanno, H., Matsuzuka, F., Narabayashi, I., Nomura, T. and Aozasa, K. (2000) Microsatellite Instability and k-ras, p53 Mutations in Thyroid Lymphoma. Japanese Journal of Cancer Research, 91, 280-286. http://dx.doi.org/10.1111/j.1349-7006.2000.tb00942.x 